\title{
ON THE SPECTRUM OF PERIODIC ELLIPTIC OPERATORS
}

\author{
JOCHEN BRÜNING AND TOSHIKAZU SUNADA
}

\section{§. Introduction}

It was observed in [Su5] that the spectrum of a periodic Schrödinger operator on a Riemannian manifold has band structure if the transformation group acting on the manifold satisfies the Kadison property (see below for the definition). Here band structure means that the spectrum is a union of mutually disjoint, possibly degenerate closed intervals, such that any compact subset of $\mathbf{R}$ meets only finitely many. The purpose of this paper is to show, by a slightly different method, that this is also true for general periodic elliptic self-adjoint operators.

Let $X$ be a Riemannian manifold of dimension $n$ on which a discrete group $\Gamma$ acts isometrically, effectively, and properly discontinuously. We assume that the quotient space $\Gamma \backslash X$ (which may have singularities) is compact. Let $E$ be a $\Gamma$-equivariant hermitian vector bundle over $X$, and $D: C^{\infty}(E) \longrightarrow \mathrm{C}^{\infty}(E)$ a formally self-adjoint elliptic operator which commutes with the $\Gamma$-action. For short, we call such a $D$ a $\Gamma$-periodic operator. It is easy to show (see Section 1 ) that the symmetric operator $D$ with the domain $C_{0}^{\infty}(E)$ is essentially self-adjoint, so that $D$ has a unique self-adjoint extension in the Hilbert space $L^{2}(E)$ of square integrable section of $E$, which we denote also by $D$ by a slight abuse of notation.

Let $C_{\mathrm{red}}^{*}(\Gamma, \mathscr{K})$ denote the tensor product of the reduced group $C^{*}$-algebra of $\Gamma$ with the algebra $\mathscr{K}$ of compact operators on a separable Hilbert space of infinite dimension, and by $\operatorname{tr}_{\Gamma}$ the canonical trace on $C_{\text {red }}^{*}(\Gamma, \mathscr{K})$. We define the Kadison constant $C(\Gamma)$ by

$C(\Gamma)=\inf \left\{\operatorname{tr}_{\Gamma} P ; P\right.$ is a non-zero projection in $\left.C_{\text {red }}^{*}(\Gamma, \mathscr{K})\right\}$.

By definition, $\Gamma$ is said to satisfy the Kadison property if $C(\Gamma)>0$. It is a conjecture proposed by Kadison that, if $\Gamma$ is torsion free, then $C(\Gamma)=1$. A

Received April 23, 1991. 
geometric example of a discrete group with the Kadison property is the fundamental group of a closed Riemann surface (cf. [P]).

THEOREM 1. If $\Gamma$ has the Kadison property, then the spectrum of any $\Gamma$-periodic elliptic operator has band structure.

In the case that the operator $D$ is bounded from below, we may establish a quantitative result on the number of intervals in the spectrum.

Theorem 2. Suppose that $D$ is a $\Gamma$-periodic elliptic operator of order $p$, and is bounded from below. Let $N(\lambda)$ be the number of components of the spectrum of $D$ which intersect the interval $(-\infty, \lambda]$. If $\Gamma$ has the Kadison property, then

$$
\limsup _{\lambda \rightarrow \infty} N(\lambda) \lambda^{-n / p} \leq C(\Gamma)^{-1} \Gamma(1+n / p) \int_{\Gamma \backslash X} A(x) d x,(n=\operatorname{dim} X),
$$

where the function $A(x)$ can be evaluated explicitly in terms of the principal symbol $\sigma D(x, \xi)$ of $D$;

$$
A(x)=(2 \pi)^{-n-1} \int_{\mathbf{R}^{n}} d \xi \int_{-\infty-\imath \gamma}^{\infty-\imath \tau} \operatorname{tr}(\sigma D(x, \xi)+i \tau)^{-1} e^{i \tau} d \tau .
$$

In Section 6, as a byproduct of our argument, we shall establish a property of the integrated density of states associated to a periodic elliptic operator.

\section{$\S 1$. Periodic elliptic operators}

Let $D: C^{\infty}(E) \longrightarrow C^{\infty}(E)$ be a $\Gamma$-periodic, formally self-adjoint elliptic operator. We shall prove that $D$ with the domain $C_{0}^{\infty}(E)$ is essentially self-adjoint. To show this, it is enough to prove that the minimal domain and maximal domain of $D$ coincide. In the case that $\Gamma$ acts freely on $X$, this is established by M. F. Atiyah [A]. The key of his proof is that $D$ has an almost local pseudo-differential parametrix $Q$, which, in the case of free actions, may be constructed by lifting an almost local parametrix on the quotient manifold $\Gamma \backslash X$. Here an operator is said to be almost local if its Schwartz kernel has support close to the diagonal. In the general case, the constrution is carried out in the following way.

Let $U_{0}, U_{1}, U_{2}$, be open sets in $X$ with $U_{0} \subset \bar{U}_{0} \subset U_{1} \subset \bar{U}_{1} \subset U_{2}$. We assume that $U_{0}$ contains the closure of a relatively compact fundamental domain for the $\Gamma$-action. One may construct an almost local pseudo-differential operator $P$ and a local smooth operator $H$ such that

$$
P D s=s+H s
$$


for $s \in C_{0}^{\infty}(E \mid U)$ and

$$
\begin{aligned}
& P\left(C_{0}^{\infty}\left(E \mid \bar{U}_{1}^{c}\right)\right) \cap C_{0}^{\infty}\left(E \mid U_{0}\right)=(0) \\
& H\left(C_{0}^{\infty}\left(E \mid \bar{U}_{1}^{c}\right)\right) \cap C_{0}^{\infty}\left(E \mid U_{0}\right)=(0) .
\end{aligned}
$$

We select $\varphi \in C_{0}^{\infty}\left(U_{0}\right)$ with

$$
\sum_{\sigma \in \Gamma} \sigma \cdot \varphi \equiv 1
$$

Since

$$
\varphi P D=\varphi+\varphi H
$$

on $C_{0}^{\infty}(E)$, putting

$$
\begin{aligned}
Q & =\sum_{\sigma \in \Gamma} \sigma(\varphi P) \sigma^{-1} \\
K_{1} & =\sum_{\sigma \in \Gamma} \sigma(\varphi H) \sigma^{-1},
\end{aligned}
$$

we have

$$
Q D=I+K_{1} .
$$

From the way of constructions, the operators $Q$ and $K_{1}$ are almost local, and $K_{1}$ is smooth. It is straightforward to see that

$$
D Q=I+K_{2},
$$

where

$$
K_{2}={ }^{t} K_{1}+D K_{1}\left({ }^{t} Q\right)-D Q\left({ }^{t} K_{1}\right),
$$

which is also almost local and smooth.

\section{§ 2. Heat kernel}

It should be noted that, to prove Theorem 1, we only have to establish the assertion for positive elliptic operators. In fact, the general case reduces to the positive case in the following manner. Suppose that the spectrum of a self-adjoint elliptic operator $D$ has a cluster at $a \in \mathbf{R}$, in the sense that there exists a sequence $\left\{a_{n}\right\}_{n=1}^{\infty}$ in $\mathbf{R}$ such that $\lim a_{n}=a$, and $a_{m}$ and $a_{n}$ lie in different components of the resolvent set of $D$ if $m \neq n$. We set, with $k \in \mathbf{N}$,

$$
D^{\prime}=\left(D-\frac{a+a_{1}}{2}\right)^{2 k}
$$




$$
a_{n}^{\prime}=\left(a_{n}-\frac{a+a_{1}}{2}\right)^{2 k}
$$

Let $\varepsilon$ be a positive number such that $\varepsilon$-neighborhood of $a_{1}$ is contained in the resolvent set. Then, for every $a_{n}$ with $\left|a-a_{n}\right|<\varepsilon, a_{n}^{\prime}$ is in the resolvent set of $D^{\prime}$, and $a_{m}^{\prime}$ and $a_{n}^{\prime}$ lie in different components of the resolvent set of $D^{\prime}$ for $m \neq n$. This implies that the spectrum of the positive operator $D^{\prime}$ has a cluster at $(a-$ $\left.\mathrm{a}_{1}\right)^{2 k} / 2^{2 k}$. Thus it suffices to handle the case of positive $D$ with order greater than the dimension of $X$. This assumption considerably simplifies our argument.

Let $D$ be a $\Gamma$-periodic self-adjoint positive elliptic operator on $X$ of order $p=$ $2 k(>n:=\operatorname{dim} X)$. We denote by $\mathscr{D}(D)$ the domain of $D$. The heat semigroup, $\exp (-t D)$, is well-defined for $t>0$. It is our first purpose to give good kernel estimates for $\exp (-t D)$, uniformly on $X$. We denote by $d$ the distance function on $X$, by $E^{*}$ the dual bundle of $E$, and by $E \otimes E^{*}$ the bundle over $X \times X$ with fiber $E_{x} \otimes E_{y}^{*}$ over $(x, y)$.

Proposition 1. Let $k(t ; x, y)$ be the kernel function for $\exp (-t D)$, which is, for $t>0$, a smooth section of $E \otimes E^{*}$. Then, for $T>0$ fixed, there are positive constants $C_{1}$ and $C_{2}$ such that

$$
|k(t ; x, y)|_{E \otimes E^{*}} \leq C_{1} t^{-n / p} \exp \left(-C_{2} t^{-1 /(p-1)} d(x, y)^{p /(p-1)}\right),
$$

uniformly for $t \in(0, T]$ and $(x, y) \in X \times X$.

we start our construction locally near an arbitrary $x_{0} \in X$.

LEMma 1. For any relatively compact connected open neighborhood $U$ of $x_{0}$ and for any $t>0$, there is a smooth operator, $F_{t}: L^{2}(E) \longrightarrow L^{2}(E)$, with a kernel function $f_{t} \in C^{\infty}\left(E \otimes E^{*}\right)$, such that the following is true.

1) $f_{t}$ depends smoothly on $t$, and $f_{t}(x, y)=0$ for $x, y \notin U$.

2) For $T>0$ fixed, there are constants $C_{3}=C_{3}(T)$ and $C_{4}=C_{4}(T)$ such that

$$
\left|f_{t}(x, y)\right|_{E \otimes E^{*}} \leq C_{3} t^{-n / p} \exp \left(-C_{4} d(x, y)^{p /(p-1)} t^{-1 /(p-1)}\right),
$$

uniformly in $0<t \leq T,(x, y) \in U \times U$.

3) For $\varphi \in C_{0}^{\infty}(X)$, with $\varphi=1$ in a neighborhood of $U$, the function $t \longmapsto$ $\varphi F_{t}$ is continuous on $[0, T]$ in the strong topology of bounded operators of $L^{2}(E)$, and $\lim _{t \rightarrow 0} F_{t} s=s$ in $L^{2}(E)$, for all $s \in L^{2}(E)$ with supp $s \subset U$.

4) For all $s \in L^{2}(E)$, the function

$$
\begin{aligned}
(0, T) & \longrightarrow \mathscr{D}(D) \\
t & \longmapsto \varphi F_{t} s
\end{aligned}
$$


is differentiable, and if we define

$$
R_{t} s:=\left(\partial_{t}+D\right) \varphi F_{t} s,
$$

then $R_{t}$ has a kernel in $C^{\infty}\left(E \otimes E^{*}\right)$, say $r_{t}$, with smooth dependence on $t>0$. Moreover, we have the estimate

$$
\left|r_{t}(x, y)\right|_{E \otimes E^{*}} \leq C_{5} \exp \left(-C_{6} d(x, y)^{p /(p-1)} t^{-1 /(p-1)}\right),
$$

uniformly in $t \in[0, T]$ and $(x, y) \in U \times U$.

5) $R_{t}$ and $D \varphi F_{t}$ are bounded in $L^{2}(E)$, and

$$
\left\|\varphi F_{t}\right\|_{L^{2}(E)}+\left\|R_{t}\right\|_{L^{2}(E)} \leq C_{7}
$$

for $t \in[0, T]$ and

$$
\left\|D \varphi F_{t}\right\|_{L^{2}(E)} \leq C_{8}(\varepsilon)
$$

for $t \in[\varepsilon, T], \varepsilon>0$.

The proof follows from [G].

To construct a global parametrix, we now choose an open connected, relatively compact set $U$ and $\varphi \in C_{0}^{\infty}(U)$ satisfying (1.1). Then it is readily seen that

$$
\lim _{t \rightarrow 0} \sigma\left(\varphi F_{t}\right) \sigma^{-1} s=s \text { in } L^{2}(E),
$$

for all $s \in L^{2}(E)$ with supp $s \subset \sigma U$, and that

$$
\left(\partial_{t}+D\right) \sigma\left(\varphi F_{t}\right) \sigma^{-1}=\sigma R_{t} \sigma^{-1} .
$$

Moreover, since the kernel functions of $\sigma\left(\varphi F_{t}\right) \sigma^{-1}$ and $\sigma R_{t} \sigma^{-1}$ are $\varphi\left(\sigma^{-1} x\right) \sigma f_{t}\left(\sigma^{-1} x, \sigma^{-1} y\right) \sigma^{-1}$ and $\sigma r_{t}\left(\sigma^{-1} x, \sigma^{-1} y\right) \sigma^{-1}$ respectively, they satisfy the estimates (2.1) (2.2).

Now the global parametrix is defined by

$$
\mathscr{T}_{t} s:=\sum_{\sigma \in \Gamma} \sigma\left(\varphi F_{t}\right) \sigma^{-1} s .
$$

We set

$$
\mathscr{R}_{t} s:=\sum_{\sigma \in \Gamma} \sigma\left(\varphi F_{t}\right) \sigma^{-1} s
$$

Lemma 2. 1) The operators $\mathscr{T}_{t}$ and $\mathscr{R}_{t}$ are continuous in $L^{2}(E)$ and

$$
\left\|\mathscr{T}_{t}\right\|_{L^{2}(E)}+\left\|\mathscr{R}_{t}\right\|_{L^{2(E)}} \leq C_{9},
$$

uniformly in $t \in[0, T]$. 
2) For $s \in L^{2}(E)$, the functions $\mathscr{T}_{t} s$ and $\mathscr{R}_{t} s$ are continuous in $t \in[0, T]$ with

$$
\lim _{t \rightarrow 0} \mathscr{T}_{t} s=s .
$$

3) $\mathscr{T}_{t} s$ is differentiable in $t \in[0, T]$, has values in $\mathscr{D}(D)$, and satisfies the equation

$$
\left(\partial_{t}+D\right) \mathscr{T}_{t} s=\mathscr{R}_{t} s
$$

Proof. Before the proof we remark that there is a constant $C_{10}$ such that, with $\varphi$ as above,

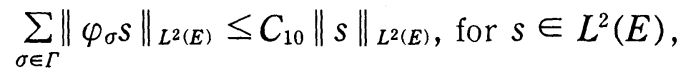

where $\varphi_{\sigma}(x)=\varphi(\sigma x)$.

1) follows easily from Lemma 1,5$)$ and $(2.6)$.

The estimate in 1) shows that the series (2.4) is uniformly convergent in $[0, T]$. By Lemma 1,3), we get 2).

It follows from Lemma 1,4$)$ that each term in the series $(2.4)$ is differentiable as a function $[0, T] \longrightarrow L^{2}(E)$, and has values in $\mathscr{D}(D)$. From Lemma 1,4$)$ and (2.6) we derive the estimate

$$
\begin{aligned}
\left\|D \mathcal{T}_{t} s\right\|_{L^{2}(E)} & \leq \sum_{\sigma \in \Gamma}\left\|D \varphi F_{t} \sigma^{-1} s\right\|_{L^{2}(E)} \\
& \leq C_{8}(\varepsilon) \sum_{\sigma \in \Gamma}\left\|\varphi_{\sigma} s\right\|_{L^{2}(E)} \\
& \leq C_{8}(\varepsilon) C_{10}\|s\|_{L^{2}(E)}
\end{aligned}
$$

uniformly in $[\varepsilon, T]$ and $s \in L^{2}(E)$, for all $\varepsilon \in(0, T)$. Now the assertion 3$)$ follows easily from Lemma 1,4 ) and 5).

Proof of Proposition 1. We apply the abstract theory of evolution equations in Banach spaces to (2.5). It is readily checked, using Lemma 2, that the assumptions of Ch. I, Theorem 6.1 in $[K]$ are satisfied. Thus we find for $t \in[0, T]$ the operator equation

$$
\exp (-\mathrm{t} D)=\mathscr{T}_{t}-\int_{0}^{t} \exp (-(t-u) D) \mathscr{R}_{u} d u
$$

Then we define again for $t \in[0, T]$,

$$
\begin{gathered}
\left(\mathscr{T} *^{0} \mathscr{R}\right)_{t}:=\mathscr{T}_{t}, \\
\left(\mathscr{T} *^{j+1 \mathscr{R}}\right):=\int_{0}^{t}\left(\mathscr{T} *^{j} \mathscr{R}\right)_{t-u} \mathscr{R} u d u, j \in \mathbf{Z}_{+} .
\end{gathered}
$$

This clearly defines continuous families of bounded operators in $L^{2}(E)$, and from 
Lemma 2,1 ) we deduce inductively the norm estimates

$$
\left\|\left(\mathscr{T} *^{j} \mathscr{R}\right)_{t}\right\| \leq C_{9}^{j+1} t^{j} / j !,
$$

On the other hand, iterating (2.7) gives for $N \in \mathbf{Z}_{+}$

$$
\exp (-t D)=\sum_{j=0}^{N}(-1)^{j}\left(\mathscr{T} *^{j} \mathscr{R}\right)_{t}+(-1)^{N+1}\left(\exp (-\cdot D) *^{N+1 \mathscr{R}}\right)_{t},
$$

which leads, with (2.8) and a similar estimate for the remainder, to the Neumann series

$$
\exp (-t D)=\sum_{j=0}^{\infty}(-1)^{\prime}(\mathscr{T} * \mathfrak{R})_{t},
$$

which is uniformly convergent for $t \in[0, T]$.

We claim that $\left(\mathscr{T} *^{j} \mathscr{R}\right)_{t}$ has kernel in $C\left(E \otimes E^{*}\right)$, for $t \in[0, T]$, with the estimate

$$
\begin{aligned}
\left|\left(\mathscr{T} *^{j} \mathscr{R}\right)_{t}(x, y)\right|_{E \otimes E^{*}} \leq & L^{j+1} C_{3}^{j+1}\left(j !\left(\begin{array}{l}
n / p \\
j
\end{array}\right)\right)^{-1}(-1)^{j} . \\
& \cdot t^{j-n / p} \exp \left(-C_{4} d(x, y)^{p /(p-1)} t^{-1 /(p-1)}\right),
\end{aligned}
$$

where

$$
L=\#\{\sigma \in \Gamma ; \sigma(\operatorname{supp} \varphi) \cap(\operatorname{supp} \varphi) \neq \phi\} .
$$

This is clear for $j=0$, by the above discussion. If $(2.10)$ is proved for some $\mathrm{j} \geq 0$, we find

$$
\begin{aligned}
& \left|\left(\mathscr{T} *^{j+1} \mathscr{R}\right)_{t}(x, y)\right|_{E \otimes E^{*}} \\
\leq & \int_{0}^{t} \int_{X}\left|\left(\mathscr{T} *^{j} \mathscr{R}\right)_{t-u}(x, y)\right|_{E^{\otimes} E^{*}} \sum_{\sigma}\left|\sigma R_{u} \sigma^{-1}(z, y)\right|_{E^{\star} E^{*}} d z d u .
\end{aligned}
$$

Now there are at most $L$ terms in the sum which are different from zero, for $y$ fixed, and for each term the volume of the support in $z$ is bounded by vol(supp $\varphi$ ), which is assumed to be one for simplicity. Hence (2.11) is obvious from the induction hypothesis, an elementary inequality for exponentials (cf. Lemma 1.4.2. in [G]), and (2.2).

Having proved (2.10), we can use the series for the kernels to obtain the estimate stated in Proposition 1.

The arguments give above also lead to the following result (cf. [G, Theorem 1.6.1]).

Proposition 2. $\operatorname{tr} k(t, x, x) \sim t^{-n / p} A(x)$ as $t \downarrow 0$, where $A(x)$ is the function defined by (0.1). 


\section{§ 3. Group $C^{*}$-algebras}

We adopt the terminology employed in [Su5].

Let $\Gamma$ be a discrete group and let $C_{\text {red }}^{*}(\Gamma)$ be the reduced group $C^{*}$-algebra of $\Gamma$. We set $C_{\text {red }}^{*}(\Gamma, \mathscr{K})=C_{\text {red }}^{*}(\Gamma) \otimes \mathscr{K}$, where $\mathscr{K}$ is the algebra of compact operators of some separable Hilbert space, say $V$. We can identify $C_{\text {red }}^{*}(\Gamma, \mathscr{K})$ with a subalgebra of

$$
\begin{gathered}
W^{*}(\Gamma, \mathscr{L})=\left\{A: L^{2}(\Gamma, V) \rightarrow L^{2}(\Gamma, V) ; A\right. \text { a bounded linear operator with } \\
A \sigma=\sigma A \text { for all } \sigma \in \Gamma\}
\end{gathered}
$$

where we regard $L^{2}(\Gamma, V)$ as a $\Gamma$-module via the right regular representation of $\Gamma$ on $L^{2}(\Gamma)$ tensored with the identity on $V$.

Let $A \in W^{*}(\Gamma, \mathscr{L})$. We define the Fourier coefficient $\widehat{A}(\sigma)$ at $\sigma$ to be a bounded operator of $V$ given by

$$
\widehat{A}(\sigma) v=\left(A \delta_{1}^{v}\right)(\sigma),
$$

where

$$
\delta_{1}^{v}(\sigma)= \begin{cases}v & \text { if } \sigma=1 \\ 0 & \text { otherwise }\end{cases}
$$

Recall that

$$
\operatorname{tr}_{\Gamma} A=\operatorname{tr} \widehat{A}(1) .
$$

Let $C_{0}^{*}(\Gamma, \mathscr{K})$ be the set of $A \in W^{*}(\Gamma, \mathscr{L})$ with $\widehat{A}(\sigma) \in \mathscr{K}$ for all $\sigma \in \mathscr{K}$ and $\widehat{A}(\sigma)=0$ for all but finitely many $\sigma \in \Gamma$. We may identify $C_{\text {red }}^{*}(\Gamma, \mathscr{K})$ with the completion of $C_{0}^{*}(\Gamma, \mathscr{K})$ with respect to the operator norm,

Lemma 3. Let $A \in W^{*}(\Gamma, \mathscr{L})$. Then

$$
\|A\| \leq \sum_{\sigma \in \Gamma}\|\widehat{A}(\sigma)\| .
$$

Proof.

$$
\begin{aligned}
\|A \varphi\|^{2} & =\sum_{\sigma}\left\|\sum_{\mu} \widehat{A}\left(\sigma \mu^{-1}\right) \varphi(\mu)\right\|^{2} \\
& =\sum_{\sigma} \sum_{\mu_{1}} \sum_{\mu_{2}}\left\langle\widehat{A}\left(\sigma \mu_{1}^{-1}\right) \varphi\left(\mu_{1}\right), \widehat{A}\left(\sigma \mu_{2}^{-1}\right) \varphi\left(\mu_{2}\right)\right\rangle \\
& =\sum_{\sigma} \sum_{\theta_{1}} \sum_{\theta_{2}}\left\langle\widehat{A}\left(\theta_{1}\right) \varphi\left(\theta_{1}^{-1} \sigma\right), \widehat{A}\left(\theta_{2}\right) \varphi\left(\theta_{2}^{-1} \sigma\right)\right\rangle \\
& =\sum_{\theta_{1}} \sum_{\theta_{2}}\left(\sum_{\sigma} \|\left\langle\widehat{A}\left(\theta_{1}\right) \varphi\left(\theta_{1}^{-1} \sigma\right) \|^{2}\right)^{1 / 2}\left(\sum_{\sigma} \|\left\langle\widehat{A}\left(\theta_{2}\right) \varphi\left(\theta_{2}^{-1} \sigma\right) \|^{2}\right)^{1 / 2}\right.\right.
\end{aligned}
$$




$$
\begin{aligned}
& \leq \sum_{\theta_{1}} \sum_{\theta_{2}}\left\|\widehat{A}\left(\theta_{1}\right)\right\|\left\|\widehat{A}\left(\theta_{2}\right)\right\|\left(\sum_{\sigma}\|\varphi(\sigma)\|^{2}\right) \\
& =\left(\sum_{\theta}\|\widehat{A}(\theta)\|\right)\|\varphi\|^{2} .
\end{aligned}
$$

COROllary. If $\widehat{A}(\sigma) \in \mathscr{K}$ for every $\sigma \in \Gamma$ and if

$$
\sum_{\sigma \in \Gamma}\|\widehat{A}(\sigma)\|<\infty
$$

then $A \in C_{\text {red }}^{*}(\Gamma, \mathscr{K})$.

Proof. Let $K_{1} \subset K_{2} \subset K_{3} \subset \cdots$ be a sequence of finite subsets in $\Gamma$ with $\cup K_{i}=\Gamma$. For an integer $N>0$, let $A_{N} \in W^{*}(\Gamma, \mathscr{L})$ be defined by

$$
\widehat{A}_{N}(\sigma)=\left\{\begin{array}{cl}
\widehat{A}(\sigma), & \sigma \in K_{N}, \\
0, & \sigma \notin K_{N} .
\end{array}\right.
$$

Then $A_{N} \in C_{0}^{*}(\Gamma, \mathscr{K})$, and $\left\|A-A_{N}\right\| \rightarrow 0$ as $N \rightarrow \infty$, so that $A \in C_{\mathrm{red}}^{*}(\Gamma, \mathscr{K})$.

\section{§ 4. Proof of Theorem 1}

Let $D$ be a positive $\Gamma$-periodic elliptic operator on $X$ of order $p$. Fix a relatively compact fundamental domain $\mathscr{F}$ in $X$ for the $\Gamma$-action, and identify $L^{2}(E \mid \mathscr{F})$ with $V$. Then we may identify $L^{2}(E)$ with $L^{2}(\Gamma, V)$, and $W^{*}(\Gamma, \mathscr{L})$ with the ring of $\Gamma$-equivariant bounded operators of $L^{2}(E)$.

We denote by $\|\sigma\|$ the word length of $\sigma$ associated with a fixed finite set of generators of $\Gamma$. It is easy to see that there exist positive constants $C_{11}$ and $C_{12}$ such that

$$
\|\sigma\| \leq C_{11} \min _{x, y \in \mathscr{F}} d(\sigma x, y)+C_{12}
$$

Since $\Gamma$ is a homomorphic image of a free group of finite rank, and a free group has exponential growth with respect to word length, we may conclude that exist constants $C_{13}$ and $C_{14}$ such that

$$
\#\{\sigma \in \Gamma ;\|\sigma\| \leq R\} \leq C_{13} \exp \left(\mathrm{C}_{14} R\right) .
$$

Lemma 4. $\exp (-D) \in C_{\text {red }}^{*}(\Gamma, \mathscr{K})$.

Proof. Let $A:=\exp (-D)$, and let $k(x, y)$ be the kernel function for $A$. The operator $\widehat{A}(\sigma)$ acting on $L^{2}(E \mid \mathscr{F})$ has the kernel function $\sigma^{-1} k(\sigma x, y)$. In view of Proposition 1 and (4.1), we have

$$
\|\widehat{A}(\sigma)\| \leq \operatorname{vol}(\mathscr{F}) \sup _{x, y \in \mathscr{F}}|k(\sigma x, y)| \leq C_{15} \exp \left(-C_{16}\|\sigma\|^{\alpha}\right)
$$


where we set $\alpha=p /(p-1)>1$. From this and (4.2), it follows that

$$
\sum_{\sigma}\|\widehat{A}(\sigma)\|<\infty,
$$

and hence $A \in C_{\mathrm{red}}^{*}(\Gamma, \mathscr{K})$ by Corollary to Lemma 3 .

The rest of proof is done in a standard manner (see[Su5]). Namely, if two real numbers $a, b$ with $a<b$ lie in the resolvent set of $D$, then, given a positive $\varepsilon$, one may find a polynomial $p(x)$ with

$$
\|(E(b)-E(a))-p(\exp (-D))\|<\varepsilon,
$$

where $\{E(\lambda)\}_{-\infty<\lambda<\infty}$ denotes the spectral resolution for $D$; i.e.

$$
D=\int_{-\infty}^{+\infty} \lambda d E(\lambda) .
$$

This implies that $E(b)-E(a) \in C_{\mathrm{red}}^{*}(\Gamma, \mathscr{K})$, and thus leads to Theorem 1 (see also the discussion in the next section).

\section{§ 5. Integrated densities of states}

Let $D$ be a self-adjoint $\Gamma$-periodic elliptic operator bounded from below, and let $\{E(\lambda)\}_{\lambda \in \mathbf{R}}$ be the spectral resolution for $D$. Note that $E(\lambda)$ is of $\Gamma$-trace class [Su5; Lemma 2]. We set

$$
\varphi(\lambda):=\varphi_{D}(\lambda):=\operatorname{tr}_{\Gamma} E(\lambda) .
$$

The function $\varphi$ is what we call the integrated density of states (cf. [Su6]). We readily check that

$$
\varphi_{D-a}(\lambda)=\varphi_{D}(\lambda+a)
$$

and if $D$ is positive, then

$$
\varphi_{D^{m}}(\lambda)=\varphi_{D}\left(\lambda^{1 / m}\right) .
$$

To prove Theorem 2, let $\lambda>0$, and let $a_{1}<a_{2}<\cdots<a_{n}$ be a sequence in the resolvent set such that $a_{i}<\lambda$ and $E\left(a_{i+1}\right)-E\left(a_{i}\right)$ is a nontrivial projection for all $i$. Since $\sum\left(E\left(a_{i+1}\right)-E\left(a_{i}\right)\right) \leq E(\lambda)$, one has

$$
(n-1) C(\Gamma) \leq \varphi(\lambda) \text {. }
$$

It remains to show the following.

PROPOSITION 3.

$$
\varphi(\lambda) \sim \lambda^{n / p} \Gamma(1+n / p) \int_{\Gamma \backslash X} A(x) d x \text { as } \lambda \uparrow \infty
$$


Proof. In view of (5.1) and (5.2), we may assume that $D$ is positive and the order $p$ is greater than the dimension of $X$.

Since

$$
\exp (-t D)=\int e^{-t \lambda} d E(\lambda)
$$

by taking the $\Gamma$-trace of both sides we obtain

$$
\operatorname{tr}_{\Gamma} \exp (-t D)=\int e^{-t \lambda} d \varphi(\lambda) .
$$

By using Proposition 2 in $\S 2$, we find

$$
\operatorname{tr}_{\Gamma} \exp (-t D)=\int_{\mathscr{F}} \operatorname{tr} k(t, x, x) d x \sim t^{-n / p} \int_{\mathscr{F}} A(x) d x
$$

as $t \downarrow 0$, from which the assertion follows by a well-known Tauberian theorem.

\section{§6. Limit formulae for densities of states}

Let

$$
\Gamma \supset G_{1} \supset G_{2} \supset \cdots
$$

be a sequence of normal subgroups in $\Gamma$ with $G_{i}$ acting freely on $X$, so that $X_{i}=$ $G_{\imath} \backslash X$ is a Riemannian manifold on which the factor group $\Gamma_{i}=\Gamma / G_{i}$ acts as a proper discontinuous isometry group. The quotient space $\Gamma_{i} \backslash X_{i}$ is naturally identified with $\Gamma \backslash X$. Let $\pi: X \longrightarrow \Gamma \backslash X$ and $\pi_{i}: X \longrightarrow X_{i}$ denote the projection maps. One may select a relatively compact fundamental domain $\mathscr{F} \subset X$ for the $\Gamma$-action such that $\pi_{\imath}: \mathscr{F} \longrightarrow \pi_{1}(\mathscr{F})$ is a diffeomorphism. It is easily seen that $\pi_{i}(\mathscr{F})$ is a fundamental domain in $X_{i}$ for the $\Gamma_{i}$-action.

We may push down the elliptic operator $D$ onto $X_{i}$, which is a $\Gamma_{i}$-periodic elliptic operator. We denote it by $D_{i}$, and put

$$
\varphi_{i}(\lambda)=\operatorname{tr}_{\Gamma_{i}} E_{\imath}(\lambda)
$$

where

$$
D_{i}=\int \lambda d E_{i}(\lambda)
$$

is the spectral resolution of $D_{i}$.

THEOREM 3. Let $D$ be a $\Gamma$-periodic self-adjoint elliptic operator bounded from below. Suppose that $\cap G_{i}=\{1\}$. Then $\lim _{i \rightarrow \infty} \varphi_{i}(\lambda)=\varphi(\lambda)$ at continuity points of $\varphi$.

Proof. Let $k_{i}(t ; p, q)$ be the kernel function for $\exp \left(-t D_{i}\right)$. Let $p=\pi_{i}(x)$ 
and $q=\pi_{i}(y)$. Then

$$
k_{i}(t ; p, q)=\sum_{g \in G_{i}} g k\left(t ; g^{-1} x, y\right),
$$

so that we obtain, using Proposition 1 again,

$$
\begin{aligned}
\int e^{-\lambda t} d \varphi_{i}(\lambda) & =\operatorname{tr}_{\Gamma_{i}} \exp \left(-t D_{i}\right) \\
& =\int_{\pi_{i}(\mathscr{F})} \operatorname{tr} k_{i}(t ; p, p) d p \\
& =\sum_{g \in G_{i}} \int_{\mathscr{F}} \operatorname{tr} g k\left(t ; g^{-1} x, x\right) d x \\
& =\int_{\mathscr{F}} \operatorname{tr} k(t ; x, x) d x+\sum_{\substack{g \neq 1 \\
g \in G_{i}}} \int_{\mathscr{F}} \operatorname{tr} g k\left(t ; g^{-1} x, x\right) d x \\
& \longrightarrow \operatorname{tr}_{\Gamma}(\exp (-t D))=\int e^{-\lambda t} d \varphi(\lambda)
\end{aligned}
$$

as $i \longrightarrow \infty$. This leads to our assertion (cf. [Su]) .

Remark. If each $G_{i}$ has finite index in $\Gamma$, then

$$
\varphi_{i}(\lambda)=\left(\# \Gamma_{i}\right)^{-1} \#\left\{\lambda_{k} ; \lambda_{k} \leq \lambda\right\},
$$

where $\lambda_{1} \leq \lambda_{2} \leq \cdots$ stands for the eigenvalues of the elliptic operator $D_{i}$ on the compact manifold $X_{i}$. Theorem 3 is a generalization of a result in [Su6].

Acknowledgement. The first author gratefully acknowledges the hospitality of Nagoya University.

\section{REFERENCES}

[A] M. F. Atiyah, Elliptic operators, discrete groups and von Neumann algebras, Astérisque, 32/33 (1976) , 43-72.

[D1] H. Donnelly, Asymptotic expansions for the compact quotients of properly discontinuous group actions, Ill. J. Mach., 23 (1979) , 484-496.

[D2] H. Donnelly, On $L^{2}$-Betti numbers for abelian groups, Canad. Math. Bull. 24 (1981) , 91-95.

[FS] D. V. Efremov and M. Shubin, Spectrum distribution function and variational principle for automorphic operators on hyperbolic spaces, Séminiore 19881989 Ecole Polytechnique, Centre de Mathematiques, Exposén ${ }^{\circ}$ VIII.

[G] P. Greiner, An asymptotic expansion for the heat equation, Arch. Rational Mech. Anal., 41 (1971), 163-218.

[KOS] T. Kobayashi, K. Ono and T. Sunada, Periodic Schrödinger operators on a manifold, Forum Math, 1 (1989), 69-79. 
[K] S. G. Krein, Linear Differential Equations in Banach Space, Trans. Math. Monographs, A.M.S. 1971.

[P] M. Pimsner, KK-groups of crossed products by groups acting on trees, Invent. Math., 86 (1986) , 603-634.

[RS] M. Reed and B. Simon, Methods of Modern Mathematical Physics, IV Analysis of Operators, Academic Press, London, 1978.

[Sh] M. A. Shubin, The spectral theory and index of elliptic operators with almost periodic coefficients, Uspechi Mat. Nauk, 34 (1979), 95-135.

[SK] M. M. Skriganov, Geometric and arithmetic methods in the spectral theory of multidimensional periodic operators, Proc. of the Steklov Inst. of Math. 171 (1985), 1-117.

[Su1] T. Sunada, Trace formula for Hill's operators, Duke Math. J., 47 (1980), $529-546$.

[Su2] - Trace formulas, Wiener integrals and asymptotics, Proc. "Spectra of Riemannian Manifolds”, Kaigai Publ., Tokyo, 1983, 103-113.

[Su3] - Unitary representations of fundamental groups and the spectrum of twisted Laplacians, Topology, 28 (1989) , 125-132.

[Su4] - Fundamental groups and Laplacians, In Proceedings of the Taniguchi Symposium on Geometry Analysis on Manifolds of the Taniguchi Symposium on Geometry and Analysis on Manifolds 1987, Springer Lecture Notes in Mathematices, 1339 (1988), 248-277.

[Su5] - Group $C^{*}$-algebras and the spectrum of a periodic Schrödinger operator on a manifold, Canad. J. Math., 44 (1992), 180-193.

[Su6] - Trace formulae in spectral geometry, Proc. ICM-90, Kyoto, SpringerVerlag, Tokyo 1991, 577-585.

J. Brüning

Institut für Mathematik

Universität Augsburg

D.8900 Augsburg

$B R D$

T. Sunada

Department of Mathematics

University of Tokyo

113 Tokyo

Japan 